\title{
TRÁNSITOS ATLÁNTICOS E INTERCONEXIONES REGIONALES EN LA ESTRUCTURACIÓN DE LOS CAMPOS HISTORIOGRÁFICOS DE LA CUENCA DEL PLATA (PRIMERA MITAD DEL SIGLO XX)
}

\author{
ATLANTIC TRAFFICS AND REGIONAL INTERCONNECTIONS IN THE STRUCTURE OF THE \\ HISTORIOGRAPHICAL FIELDS OF THE Río DE LA PLATA BASIN (FIRST HALF OF THE 20TH CENTURY) \\ Tomás Sansón Corbo* \\ slbt@hotmail.com
}

RESUMEN: Las transformaciones políticas, socioculturales y económicas acaecidas en el entorno de 1900 en los países de la Cuenca del Plata afectaron los contextos de producción de conocimiento histórico. Aunque subsistieron los vínculos interpersonales, las redes intelectuales adquirieron gradualmente características más formales. En esta ponencia pretendo estudiar los fenómenos de intercambio, complementariedad y tránsito de influencias teórico-metodológicas, gestionados por agentes diversos (algunos de países de la región y otros de origen europeo) durante la primera mitad del siglo XX. Focalizo el análisis en los aportes de especialistas extranjeros en centros locales de formación superior en Historia. Analizo en particular los casos de Rafael Altamira en Argentina, Fernand Braudel en Brasil, Guy de Hollanda en Paraguay, Emilio Ravignani y José Luis Romero en Uruguay. El objetivo es valorar, desde una perspectiva comparada, el influjo de tales contribuciones en la configuración de los campos historiográficos nacionales.

Palabras ClaVE: Historiografía comparada, Profesionalización de la Historia, Cuenca del Plata.

ABSTRACT: The political, sociocultural and economic transformations which took place around 1900 in the countries of the Río de la Plata Basin affected the contexts of production of historical knowledge. Although the interpersonal ties survived, the intellectual networks acquired gradually more formal features. In this paper I try to study the phenomena of exchange, complementarity and traffic of theoretical-methodological influences, managed by diverse agents (some from countries of the region and others from European origin) during the first half of the 20th century. I focus the study on the contributions of foreign specialists in local centers of top formation in History. I analyze specially the cases of Rafael Altimara in Argentina, Fernand Braudel in Brazil, Guy de Hollanda in Paraguay, Emilio Ravignani and José Luis Romero in Uruguay. The aim is to value, from a comparative perspective, the influence of such contributions for the configuration of the national historiographical fields.

KEYWORDS: Comparative historiography, Professionalization of History, Río de la Plata Basin.

\section{Presentación}

La evolución de los estudios históricos en los Estados de la región platense estuvo condicionada, entre otros factores, por la acción de prestigiosos historiadores extranjeros contratados por las universidades locales con el propósito de organizar y dinamizar la

\footnotetext{
*Doctor en Historia por la Universidad Nacional de La Plata (Argentina). Profesor e investigador de la Facultad de Humanidades y Ciencias de la Educación de la Universidad de la República (FHCE-UDELAR, Uruguay). Integrante del Sistema nacional de Investigadores de la Agencia Nacional de Investigación e Innovación (SNIANII, Uruguay).
} 
formación de investigadores profesionales. Los casos más significativos fueron los de Rafael Altamira en la Universidad de la Plata (1909), Fernand Braudel y la "misión francesa" en San Pablo (1935-1937), Emilio Ravignani y José Luis Romero en Montevideo (1947) y Guy de Hollanda en Asunción (1948). Esos intelectuales debieron insertarse en medios académicos que estaban, en la mayoría de los casos, en proceso de estructuración y actuar sobre tradiciones historiográficas con diferentes niveles de consolidación.

La contratación de referentes extranjeros y la existencia de ámbitos institucionales y formativos fungieron como espacios efectivos de interfaz entre las demandas endógenas y los acicates exógenos. En esos ámbitos se articularon las sinergias que posibilitarían la configuración, con diversidad de ritmos, de los campos historiográficos nacionales.

\section{Tránsitos atlánticos}

\subsection{Rafael Altamira en la Universidad Nacional de La Plata (Argentina)}

En la encrucijada de los siglos XIX y XX la historiografía argentina experimentó una serie transformaciones. Una de las más significativas fue la profundización de los procesos de institucionalización y profesionalización de los estudios históricos.

La formación de historiadores profesionales fue posible a partir de la fundación de la Facultad de Filosofía y Letras de la Universidad Nacional de Buenos Aires $(1896)^{1}$ y de la Facultad de Humanidades y Ciencias de la Educación de la Universidad Nacional de La Plata (1920). Emilio Ravignani y Ricardo Levene, respectivamente, fueron sus referentes fundamentales desde la década de 1920.

Joaquín V. González, destacado jurista y exponente "de las corrientes reformistas de la elite argentina" (BUCHBINDER, 2005, p. 81) de principios del siglo XX, impulsó en la ciudad de La Plata un proyecto universitario renovador. Planificó una universidad moderna. ${ }^{2}$

\footnotetext{
${ }^{1}$ En 1905 se creó la Sección Historia de la Facultad de Filosofía y Letras. Entre sus objetivos estaba organizar y publicar las investigaciones realizadas en el marco de la Facultad y realizar relevamientos de fuentes con el propósito de escribir una historia nacional. En 1911 comenzó la edición de las primeras series documentales. Emilio Ravignani adquirió un fuerte protagonismo. En 1920 fue designado director de la Sección que, al año siguiente, se transformó en Instituto de Investigaciones Históricas.

${ }^{2}$ La nueva institución se caracterizó por otorgarle a los docentes un rol fundamental en el gobierno y en la administración; asignarle a la Facultad de Ciencias Jurídicas y Sociales la responsabilidad de formar no sólo abogados, sino de propender al desarrollo de los ciencias sociales; establecer como prioridad las relaciones entre la enseñanza media y la superior (iniciativa que se concretó, entre otras medidas, con la creación de un Colegio Nacional dependiente de la Universidad); enfatizar las actividades de extensión como tarea
} 
Implementó su proyecto a partir de 1906 cuando fue elegido Presidente de la Universidad (cargo en el que permaneció hasta 1918). Una de sus preocupaciones fue promover contactos e intercambios con intelectuales y centros universitarios europeos con el propósito de fortalecer las diferentes áreas académicas de la joven institución. Gracias a esa política proactiva fue posible gestionar la visita de Rafael Altamira. ${ }^{3}$

Altamira era un destacado intelectual español vinculado con el krausismo y el "institucionismo", corrientes que propugnaban un "esfuerzo de racionalización, de educación, de modernización, de secularización" (PELOSI, 2012, p. 15) y que tenían uno de sus principales centros de irradiación en la Universidad de Oviedo. Compartía con muchos de sus contemporáneos la necesidad de impulsar una modernización social y pedagógica que permitiera a España superar el impacto producido por las pérdidas de los territorios coloniales. Su pensamiento y producción contribuyeron a "disipar una visión negativa de la colonización española", su "vindicación de la acción civilizadora de España en América formó parte de su nacionalismo historiográfico" (PELOSI, 2012, p. 17).

Arribó a Argentina en 3 julio de 1909 y permaneció hasta el 27 octubre. ${ }^{4}$ Desarrolló una intensa actividad académica que tuvo como epicentro la Sección de Filosofía, Historia y Letras de la UNLP. ${ }^{5}$ También dictó conferencias y cursos sobre temas diversos en las facultades de Filosofía y Letras y de Derecho de la Universidad de Buenos Aires y en centros académicos de Santa Fe, Córdoba y Rosario. Realizó, además, una fugaz visita a Montevideo entre el 4 y el 12 de octubre.

La acción de Altamira tuvo, según Gustavo Prado, importantes repercusiones en la opinión pública y en las élites letradas y dirigentes. Resultó además, muy oportuna en el marco de una sociedad en transformación que se aprestaba a celebrar el centenario de los hechos de mayo de 1810. Su prédica hispanista era atractiva para la "fracción reformista de la elite", interesada "en la apertura social y la democratización del sistema oligárquico"

\footnotetext{
fundamental para influir "en aquellos sectores de la sociedad que no estaban en condiciones de acceder a los estudios regulares que se llevaban a cabo en las aulas" (BUCHBINDER, 2005, p. 83).

${ }^{3}$ No estudiaré en esta instancia las presencias de Lucien Febvre (1937) y Fernand Braudel (1947) en Buenos Aires debido a que las influencias de las mismas se efectivizaron en la década de 1950, etapa que está fuera del período de referencia.

${ }^{4}$ Fue la primera escala de un largo viaje que culminaría en marzo de 1910 y le permitió visitar Uruguay, Chile, Perú, México y Cuba.

${ }^{5}$ Sección recientemente organizada en el seno de la Facultad de Ciencias Jurídicas y Sociales, mojón fundamental del proceso que culminaría en 1920 con el establecimiento de la Facultad de Humanidades y Ciencias de la Educación.
} 
(PRADO, 2013, p. 140). Surgió un "nuevo hispanismo" en Argentina, impulsado por la comunidad española local, y muy bien recibido por los sectores dirigentes que miraban con reticencia el fenómeno de la presencia mayoritaria de inmigrantes no hispanos (70\%) que hacía "temer por la disolución de la identidad rioplatense" (PRADO, 2013, p. 142).

Uno de sus aportes más fecundos en la universidad platense los realizó en un curso dedicado específicamente a la Metodología de la Historia. ${ }^{6}$ Su insistencia

[...] en la preparación, crítica y aprovechamiento de fuentes constituía una de las columnas vertebrales de su teoría, inspirada en la historiografía alemana. Los autores citados eran Ernst Bernheim y Charles Seignobos y siguiendo a este último afirmó la constatación de los hechos, en similitud con lo que realiza el científico a través del microscopio. Sin embargo, en una actitud vanguardista, según Altamira lo histórico no era sólo lo escrito [...] aunque conserve las normas críticas enunciadas por Ranke, insistió en que la historia debía ser historia de la civilización (PELOSI, 2005, p. 29-30).

Dedicó tiempo a estudiar los programas de enseñanza de la historia argentina en todos los niveles del sistema. Formuló recomendaciones para transformarla y optimizarla. Sugirió priorizar la historia americana y la argentina con perspectivas patrióticas para viabilizar una verdadera "regeneración nacional" (PELOSI, 2005, p. 32). Ricardo Levene tomaría seriamente tales consejos.

Otro de los ejes de la acción de Altamira fue la extensión. Participó en actividades culturales con trabajadores y mostró total sintonía con los proyectos reformistas de González, para quien "la mayor ilustración del obrero y los sectores populares eran, en cierta medida, un elemento que contribuía de manera decisiva a la cohesión social" (BUCHBINDER, 2005, p. 84).

La presencia Altamira en Argentina fue breve, pero se produjo en una etapa clave de su desarrollo historiográfico: la transición entre las prácticas decimonónicas y la renovación motorizada por la Nueva Escuela Histórica. Su mayor aporte "consistió en respaldar una praxis historiográfica comprometida con un ideal científico a través de un ejercicio de clarificación y fundamentación de las técnicas y utillajes del oficio del historiador" (BUCHBINDER, p. 146). Sus enseñanzas fueron complementadas con un magisterio "no presencial" ejercido mediante artículos, libros y el contacto epistolar que

\footnotetext{
${ }^{6}$ El curso estaba basado en sus obras sobre La enseñanza de la historia (1891) y Cuestiones modernas de historia (1904).
} 
mantuvo por décadas con intelectuales como Juan Alvarez, Luis Ma. Torres, Rómulo Carbia y, muy especialmente, Ricardo Levene. ${ }^{7}$

\subsection{Fernand Braudel y la "misión francesa" en la Universidad de San Pablo (Brasil)}

Durante el período de la "vieja república", la historiografía brasileña experimentó un período de transición. Hasta la década de 1920 la orientación general de los estudios históricos tuvo una impronta asociativa y academicista cuyo paradigma era el Instituto Histórico y Geográfico de Brasil. ${ }^{8}$ Paulatinamente, las universidades pasaron a tener preeminencia en la gestión de la indagatoria sobre el pasado.

El Estado surgido "de la Revolución de 1930 pasó a invertir en educación superior para la formación de cuadros y para el debate más profundo de los problemas de Brasil" (REIS, 2007, p. 117). Las clases medias comenzaron a acceder a la enseñanza superior y en particular al aprendizaje de las humanidades y de la historia cuya práctica, hasta entonces, estaba mayoritariamente reservada a miembros de los institutos históricos y geográficos. Para responder a los desafíos y preguntas planteados por los grandes problemas que aquejaban al país los historiadores recurrieron a estudios sociales, económicos y culturales, necesariamente transitaron por la vía de la inerdisciplinariedad.

Al advenimiento del varguismo existían solamente dos instituciones universitarias relativamente consolidadas, la de Río de Janeiro y la de Mina Gerais, creadas en 1920 y 1927 respectivamente (TEIXEIRA LOPES, MENDES FARIA FILHO, GREIVE VEIGA, 2015, p. 161166). En ninguna de ellas existían cursos de carácter humanístico. La situación cambió en 1934 con la fundación de la Facultad de Filosofía, Ciencias y Letras de la Universidad de San Pablo.

Tras la derrota de la Revolución Constitucionalista (1932), las élites paulistas impulsaron un interesante movimiento cultural con el propósito de compensar la hegemonía

\footnotetext{
${ }^{7}$ El influjo del intelectual español sobre las prácticas y criterios historiográficos de Ricardo Levene se expresó concretamente en: el interés por el derecho indiano; la preferencia por establecer vínculos intelectuales, editoriales y de intercambio con autores e instituciones españolas; la necesidad de incursionar en una nueva historia pautada por temáticas sociales y económicas, complemento necesario de los tradicionales enfoques políticos y militares; la preocupación por la enseñanza de la historia en cuanto agente de formación de la conciencia nacional y ciudadana; la extensión como función universitaria fundamental para amplificar en la sociedad los bienes de la cultura.

${ }^{8}$ El modelo institucional se vio fortalecido por la creación y/o dinamización de centros provinciales en Pernambuco, Ceará y Bahía -inspirados en el referente carioca- que contribuyeron a dinamizar los estudios históricos regionales.
} 
política que habían perdido en la Federación (CORRÊA LIMA, 2009, p. 67-68). El periódico O Estado de São Paulo se transformó en propagandista de la iniciativa y el periodista Júlio Mesquita Filho en su principal promotor. Por decreto estadual del 25 de enero de 1934 se instituyó la Universidad de San Pablo.

El educador Fernando de Azevedo fue uno de los principales impulsores de la Facultad de Filosofía. La concibió como un "centro de formación de profesores para la enseñanza secundaria" y un ámbito de "estudios de cultura libre y desinteresada", verdadero "corazón de la Universidad" (TEIXEIRA LOPES, MENDES FARIA FILHO, GREIVE VEIGA, 2015, pp. 161-168). Se procuraba sustituir la mera transmisión de información y datos por el aprendizaje de habilidades didácticas y metodológicas tendientes a la creación de conocimiento. Para concretar tales objetivos y para convertirla en un centro de excelencia, en el que se formaría la inteligentzia paulista, se consideró necesario contar con el concurso de docentes europeos. Durante el primer año de funcionamiento hubo gestiones que culminaron con la contratación de tres profesores alemanes, cuatro italianos y seis franceses de diversas disciplinas.

Los vínculos culturales entre Francia y Brasil eran muy fuertes desde el siglo XIX. Estuvieron favorecidos por organismos como el "Grouppement des Universités et Grandes Ecoles de France pour le Développement des Relations avec l'Amérique Latine" (creado en 1908). Las "misiones" científicas francesas fueron posibles gracias a la firma de un acuerdo oficial entre el gobierno paulista y el francés.

Fernand Braudel participó en la misión francesa -integrada entre otros por LéviStrauss, Roger Bastide y Pierre Verger- que colaboró a partir de febrero de 1935 en la organización de la Facultad de Filosofía, Letras y Ciencias. Fue contratado para hacerse cargo de la cátedra de Historia de las Civilizaciones. Permaneció en Brasil hasta 1937. Encontró un país joven y pujante, una sociedad en formación que le hizo reflexionar y estimuló su creatividad. En cierta ocasión confesó: "Yo me torné inteligente yendo a Brasil" (CORRÊA LIMA, 2009, p. 15).

El historiador francés desarrolló una intensa actividad docente. Dictó conferencias y publicó algunos artículos. Estableció vínculos perdurables con el filósofo João Cruz Costa y el periodista Júlio Mesquita Filho. Actuó, además, como puente entre éstos y el medio académico europeo. Facilitó contactos y redes de intercambio. Contribuyó, además, a 
difundir en Francia la obra de Gilberto Freyre. Uno de sus mayores aportes, desde la perspectiva metodológica, fue proponer nuevos tipos de pesquisa a partir de la formulación de problemas de investigación. Formó un grupo de historiadores y docentes que posteriormente lo reconocerían como maestro. Con algunos alumnos como Alice Canabrava, Branca Caldeira y Eurípedes Simões de Paula, estableció lazos más personales. Era frecuente que los invitara a su casa para orientarlos o continuar el examen de cuestiones que quedaban pendientes en el aula.

La influencia de la "misión francesa" fue muy importante. Impulsó la realización y difusión de obras y formas de trabajo que ya se venían cultivando. Desde comienzos del siglo XX los intelectuales brasileños habían tendido a centrarse en cuestiones relacionadas con la identidad nacional; autores como Euclides da Cunha y Paulo Prado, entre otros, procuraron explicar -en sendas obras de carácter ensayístico e interdisciplinario como Os Sertões (1902) y Retrato do Brasil (1926), respectivamente- la naturaleza profunda y la esencia sociocultural de Brasil. La producción de Gilberto Freyre (1900-1987) ${ }^{9}$, Caio Prado Junior (1907-1990) ${ }^{10}$ y Sergio Buarque de Holanda (1902-1982), los "intérpretes de Brasil”, demuestra que había un campo favorable para la recepción de la historiografía braudeliana.

Braudel volvió a San Pablo en 1947. Entre mayo y diciembre dictó cursos en la Facultad. Viajó, además, por Argentina y Chile.

Uno de los frutos más duraderos de la acción francesa en San Pablo fue la creación de la Revista de História (1950). Fundada por Eurípides Simões de Paula y patrocinada por Braudel, se transformó en una de las publicaciones académicas más prestigiosas de Brasil.

\footnotetext{
${ }^{9}$ Uno de los mejores ejemplos para probar lo expuesto es Casa grande \& senzala, de Freyre, editada en 1933. Es un trabajo que presenta enfoques y temas que serían muy caros para la Escuela de los Anales como la religiosidad popular y la sexualidad; cuestiones encaradas desde una perspectiva histórico-antropológica inspirada en el alemán Franz Boas, autor de Anthropology and modernlife (1929), de quien fue alumno en la Universidad de Columbia. Libro pionero y anterior al desembarco paulista de los franceses -en el que palpitan formas de encarar la reconstrucción del pasado como la historia de las mentalidades y el abordaje interdisciplinario que serían posteriormente sello de distinción de los Annales- que Braudel se encargaría de difundir a nivel internacional. Mientras estaba en prisión, durante la segunda guerra mundial, Braudel escribió en Annales (1943) una reseña sobre la obra de Freyre.

${ }^{10}$ Caio Prado frecuentó las clases de Braudel, Roger Bastide y Pierre Monbeig. El contacto con ellos contribuyó "para tornar más concreta la búsqueda de la diversidad del espacio y del tiempo en su elaboración marxista de la historia"; la geografía, en particular, coadyuvó "decisivamente para la habilidad con que describía mediaciones sociales" y fue, además, uno "de sus recursos para señalar las formas intermediarias que el sistema colonial fue adquiriendo en el proceso de poblamiento" (D'INCAO, 1989, p. 384).
} 
La acción de la misión francesa en San Pablo fue coetánea a una serie de procesos y fenómenos que coadyuvaron a consolidar el campo historiográfico brasileño (ampliación del mercado editorial ${ }^{11}$, apoyo explícito del gobierno a las indagaciones relacionadas con la identidad nacional, fundación de instituciones universitarias dedicadas a la formación de docentes e investigadores, continuidad de la labor de instituciones como el IHGB, conformación de comunidades historiográficas que competirían entre sí, abordajes interdisciplinarios, interés por temas económicos, sociales y culturales). La indagatoria del pasado se transformó en una actividad profesional ejercida en centros de investigación. La titulación se convirtió en un requisito de legitimación.

Los "alumnos de Braudel"12 contribuyeron a la renovación general de la historiografía brasileña. ${ }^{13}$ Las tesis de doctorado presentadas y defendidas a partir de 1940 en la Facultad de Filosofía de la USP reflejan la incidencia de los nuevos enfoque teóricos y metodológicos aportados por los profesores franceses. ${ }^{14}$

\section{Interconexiones regionales}

\subsection{Emilio Ravignani y José Luis Romero en la Universidad de la República (Uruguay)}

En Uruguay, los antecedentes de la enseñanza universitaria de la Historia se remontan a comienzos del siglo XX con los cursos de la Cátedra de Derecho Constitucional en la Facultad de Derecho de la Universidad de la República. Entre 1935 y 1945 funcionó en la órbita de esa cátedra, un Seminario de Derecho Indiano regenteado por Eugenio Petit

\footnotetext{
${ }^{11}$ Hubo un incremento de la producción intelectual que se expresó en un boom editorial de obras de ficción, traducciones de libros extranjeros, trabajos didácticos vinculados a la realidad brasileña. Se multiplicaron las editoriales y la edición de colecciones seriadas, entre las que se destacan: Coleção Brasiliana (San Pablo, 1931), Documentos Brasileiros (Río de Janeiro, 1936), Biblioteca Histórica Brasileira (San Pablo, 1940) (cf. LAUERHASS, NAVA, 2007, p. 89).

12 En 1948 apareció en Annales un número dedicado a América Latina con varias reseñas de obras de discípulos brasileños de Braudel. Se destacan en particular los comentarios sobre el trabajo de Alice Canabrava.

13 Florestan Fernandes, por ejemplo, considera que "los profesores franceses no nos trajeron la civilización moderna, pero nos colocaron más próximos del centro de producción intelectual más fecundo de esa civilización” (D'INCAO, 1989, p. 28). Eurípides evocaba en 1971, con tono reverencial, la pléyade de historiadores franceses que actuaron en San Pablo en la década de 1930 y formaron a su generación en la USP (SIMÕES DE PAULA, 1971, p. 430). Valoraba la interdisciplinariedad practicada por los historiadores brasileños (con la Geografía, la Sociología, la Economía Política, la Filosofía, entre otras ciencias sociales) como uno de sus efectos más importantes.

14 Las transformaciones fueron particularmente evidentes en las tesis de Eurípedes Simões de Paula $(O$ comércio varegue e o Grão-Principado de Kiev, 1942), Alice Canabrava (O comércio no Rio da Prata de 1580 a 1640, 1942), Astrogildo Rodrigues de Mello (A política colonial de Espanha a través das Encomiendas, 1942) y Eduardo d'Oliveira França (A realeza em Portugal na Idade Média e as origens do absolutismo, 1945).
} 
Muñoz. Fue el primer espacio universitario dedicado a la investigación histórica; algunos de los participantes del mismo integrarían posteriormente los cuadros docentes y estudiantiles de la Facultad de Humanidades y Ciencias.

Durante el rectorado de Carlos Vaz Ferreira (1935-1939) se crearon seis cátedras vinculadas a la formación en ciencias naturales y humanas; la de Historia funcionó en régimen de seminario, a cargo de Lincoln Machado Ribas, fue uno de los pilares sobre los que se organizaría el Instituto de Investigaciones Históricas de la Facultad de Humanidades y Ciencias. ${ }^{15}$

A comienzos de la década de 1940 existía en el país un clima favorable para la creación de un centro superior de enseñanza de la Historia. El contexto era complejo, había profundas divisiones en la sociedad (motivadas por el golpe de Estado de Gabriel Terra, los alineamientos ideológicos generados durante la Guerra Civil Española y la Segunda Guerra Mundial, el establecimiento del peronismo en Argentina) que se prolongaron en el tiempo y tuvieron proyección en la corporación de historiadores. En el imaginario colectivo predominaba una convicción sintentizada en la fórmula: "golpista=falangista=nazifascista=peronista" (ZUBILLAGA, 2002, p. 166). Esta "ecuación ideológica" contribuye a entender ciertos hechos y enfrentamientos acaecidos en la etapa de surgimiento de la Facultad de Humanidades (1945) y del Instituto de Profesores Artigas (1949).

La Facultad se creó por ley en octubre de 1945. El filósofo Carlos Vaz Ferreira tuvo un rol destacado en todo el proceso. La institución funcionó al principio como un conglomerado de cátedras, sin planes de estudio definidos. Incorporó a sus cuadros docentes a intelectuales europeos que habían sido desplazados por el fascismo.

Entre los docentes y estudiantes de las diversas disciplinas surgió la intención de organizar los estudios. Los de Historia, en particular, plantearon ante las autoridades la idea de crear un Instituto de Investigaciones similar al homónimo porteño y propusieron a Emilio Ravignani como posible director. El Consejo de la Facultad trató el tema e inició gestiones oficiales con Ravignani. Este aceptó el ofrecimiento pues vio en esa oferta la posibilidad de continuar sus actividades académicas que se habían resentido desde el advenimiento del peronismo.

\footnotetext{
${ }^{15}$ Cf.: PARIS DE ODDONE, 1995; ZUBILLAGA, 2002.
} 
La designación de Ravignani no estuvo exenta de conflictos. Motivó protestas en el Parlamento y la prensa formuladas principalmente, aunque no exclusivamente, por adherentes al Partido Nacional. Se cuestionó el hecho de confiar a un extranjero (un "porteño") el estudio del pasado nacional y la exclusión de Juan Pivel Devoto, considerado candidato natural para el cargo. Debe tenerse en cuenta que existían en torno a Pivel sospechas de cierto filonazismo y simpatías por el régimen de Francisco Franco (ZUBILLAGA, 2002, p. 76-83) que lo inhibían -en opinión de quienes tenían la responsabilidad de elegir al director del Instituto- para ocupar el cargo. Ravignani era considerado un candidato más potable debido a su reconocida militancia antifascista y opositora al gobierno argentino (que encuadraba muy bien con el mayoritario sentimiento antiperonista de la sociedad uruguaya).

El Instituto de Investigaciones se inauguró oficialmente el 6 de octubre de 1947 y funcionó a partir de entonces bajo la orientación de Ravignani. Durante su gestión (1947 a 1954) contribuyó a renovar las prácticas tradicionales. Aplicó los criterios heurísticos, didácticos y epistemológicos que había implementado en la UBA. Estableció relaciones con centros académicos de Europa, Estados Unidos y América Latina, propuso un plan editorial para canalizar la publicación de fuentes, estableció programas y proyectos de investigación abiertos a indagatorias y contrastaciones empíricas. Fomentó la instalación de "delegaciones" del Instituto de Investigaciones Históricas en Buenos Aires, Sevilla, Londres y París, con el objeto de relevar documentación custodiada en repositorios de esas ciudades. La Universidad de la República lo distinguió con el título de Doctor Honoris Causa en diciembre de 1952.

José Luis Romero fue otro de los referentes de la etapa inicial de la Facultad de Humanidades. En 1949 fue designado docente de dos materias claves de la Licenciatura de Historia: "Introducción a los Estudios Históricos" y "Filosofía de la Historia". Contribuyó a superar el modelo neopositivista vigente y propició un acercamiento del "estudiantado de Historia a la teoría del conocimiento, sorteando de tal modo el escollo generalizado de una enseñanza que oscilaba ente lo puramente fáctico y la interpretación ‘impresionista` del pasado" (ZUBILLAGA, 2002, p. 183). A partir de 1952 su labor estuvo centrada en el "Seminario de Historia de la Cultura", espacio académico renovador, que estimuló el acercamiento de los estudiantes a las fuentes originales de conocimiento y la reflexión 
crítica sobre las mismas. El Seminario dio lugar en 1962 a la creación de la Sección Historia de la Cultura, dirigida honorariamente por Romero quien realizaba periódicas visitas a Montevideo. Bajo su orientación se formaron historiadores de la talla de Juan Antonio Oddone y Gustavo Beyhaut.

En la década de 1950 egresaron de la Facultad las primeras promociones de profesionales de la historia, entre los que se destacaron Juan Oddone y Blanca París. Estos debieron competir en el incipiente campo historiográfico con los graduados del IPA, institución coetánea fundada en 1949, que tenía como propósito fundamental formar docentes para la Enseñanza Media.

\subsection{Guy de Hollanda y la "misión brasileña" en Paraguay}

Si bien los antecedentes de la profesionalización de los estudios históricos en Paraguay se remontan a mediados de la década de $1930^{16}$, fue en 1944 cuando el gobierno de Higinio Morínigo creó una Escuela Superior de Humanidades con el objetivo de "otorgar un título de nivel superior a los maestros y maestras que habían culminado sus estudios en la Escuela Normal de Profesores" (DE LOPEZ MOREIRA, 2012, p. 42). La institución comenzó sus actividades en junio. Concurrieron a sus aulas decenas de jóvenes docentes que deseaban perfeccionar sus conocimientos. Contó, en el período inicial, con el apoyo de la Embajada de Brasil y de la Misión Cultural Brasileña. Esto le permitió contratar profesores de ese origen para fortalecer al cuerpo docente nacional. Uno de los más importantes en el área de Historia fue Guy de Hollanda.

El origen de esta colaboración nació en ocasión de la visita de Getulio Vargas a Paraguay (GAMARRA, 2014, p. 2) y en el contexto general de la secular puja entre Argentina y Brasil por la hegemonía en la Cuenca del Plata. En la década de 1940 decayó la influencia

${ }^{16}$ El Dr. Justo Prieto (Ministro de Educación de Paraguay) planteó 1934, en el marco de la II Conferencia Interamericana de Educación que se desarrollaba en Santiago de Chile, la creación de una Facultad Libre de Humanidades; la idea respondía a la necesidad de preparar docentes para la enseñanza media; el contexto bélico no permitió la implementación del proyecto. Fue necesario esperar hasta 1939, cuando el Presidente Félix Paiva creó -por Decreto-Ley no 14.836, del 21 de junio- la Facultad Libre de Humanidades; el Consejo Superior Universitario debatió por largo tiempo los planes de estudio, los cursos comenzaron recién en 1943, pero el centro funcionó solamente dos años. Paralelamente las autoridades del Ateneo Paraguayo presentaron, el 7 de marzo de 1941, una solicitud al Rector de la UNA para crear una Facultad de Humanidades; las complicaciones institucionales, la existencia de una institución similar en trámite de implementación y las carencias presupuestales determinaron que el proyecto fracasara. En 1944 el gobierno autorizó al Centro Cultural Paraguayo-Americano (institución privada dedicada a la enseñanza del idioma inglés) a fundar un Instituto Libre de Humanidades que funcionó por tres años (DE LOPEZ MOREIRA, 2012). 
económica y cultural ejercida por Argentina. La diplomacia brasileña -respondiendo a una estrategia propagandística del gobierno de Vargas, que buscaba proyectar en América Latina la lengua portuguesa y la cultura brasileña ${ }^{17}$ - paulatinamente se fue imponiendo. Hitos fundamentales de este proceso fueron las visitas de Vargas a Asunción (1941) y la de Higinio Morínigo a Río de Janeiro (1943). Mejoraron notoriamente las relaciones entre ambos Estados. Se firmaron varios convenios referidos a cuestiones fronterizas, comerciales y culturales. Aumentó la cooperación mutua. En ese contexto se creó, en mayo de 1943, el Instituto Cultural Paraguay-Brasil, conocido como “Misión Cultural Brasileña". 18 Tenía por objetivo "estrechar y fomentar el intercambio cultural, científico y educativo entre Paraguay y Brasil" (GARCÍA, 2014). Esta cooperación adquirió mayor impulso con la firma de un acuerdo en marzo de 1952 que, entre otros asuntos, fortalecía la colaboración con la UNA facilitando el intercambio docente, científico y artístico.

La Escuela Superior de Humanidades fue la base de la Facultad de Filosofía de la UNA. La institución fue creada por Decreto-ley no 24.929, del 16 de febrero de 1948, del Presidente Morínigo. Surgió como resultado del esfuerzo y gestiones conjuntas realizadas ante las autoridades nacionales por intelectuales vinculados a la Escuela Superior -y otros ajenos a la misma- que consideraban necesario jerarquizar los estudios humanísticos. Estaba concebida como un centro superior de preparación de docentes, formación de investigadores y promoción de la cultura nacional. Se trataba de aspiraciones nobles de dudosa concreción en lo inmediato. Los avatares políticos determinaron que al mes siguiente, el 9 de marzo, el Presidente decretara la intervención de la Universidad.

El Dr. Juan Vicente Ramírez fue designado decano. Las actividades se inauguraron el 10 de abril de 1948 en el marco de un sencillo acto. El discurso de Ramírez fue muy significativo porque refleja el fuerte encorsetamiento en que se desarrollaría la labor humanística:

\footnotetext{
17 “A fines de la década del 50, estos programas habían sido implantados en cuatro ciudades sudamericanas: el primero fue en Montevideo en 1940; el segundo en Asunción en 1943, luego Buenos Aires en 1954 y posteriormente en La Paz en 1958" (GARCÍA, 2014).

18 No resulta claro establecer la "distinción entre los objetivos y funciones del Instituto Cultural Paraguay Brasil y la Misión Cultural Brasileña". Aparentemente "coexistieron con atribuciones relativamente comunes y paulatinamente la Misión fue sustituyendo al Instituto. Sin embargo, en las notas de prensa contemporáneas ambas denominaciones devinieron en sinónimos para designar a la burocracia cultural brasileña" (GARCÍA, 2014).
} 
Si me dirijo a ustedes en este momento es sólo para presentarles mi cordial saludo e invitarles a meditar el significado de este minuto solemne en que bajo nuestro cuidado y responsabilidad, inicia sus primeros pasos la Facultad de Filosofía. Todos comprenderán nítidamente que es de absoluta necesidad que estos pasos iniciales los realice con acierto y felicidad, porque de ellos dependerá en gran manera su destino. Si los da con fortuna, tiene asegurado el porvenir, ya que con ellos se irá formando la tradición que facilitará para ella el advenimiento de días llenos de luz y de gloria. En cambio, si comienza a andar por senderos equivocados, costará doble esfuerzo para reparar el error y continuar después por el buen camino (DE LOPEZ MOREIRA, 2012, p. 48).

Tanto la enseñanza como la investigación estaban bajo sospecha $y$, por ende, limitadas en su ejercicio. El contexto político conspiró contra el normal funcionamiento de la nueva Facultad. Fue así durante el período de inestabilidad institucional que se inició con la caída de Morínigo (3 de junio de 1948), prosiguió en el gobierno de Federico Chaves (11 de setiembre de 1949 al 4 de mayo de 1954) y se agudizó a partir de la asunción del General Alfredo Stroessner (15 de agosto de 1954 al 3 de febrero de 1989).

La heteronomía académica contribuyó al aherrojamiento del saber, favoreció la inercia epistemológica y entorpeció el surgimiento de un campo disciplinario. No existían condiciones para la producción original y crítica de conocimiento. Parecía difícil concretar en lo inmediato el deseo del decano de que la Facultad ganara "el mismo prestigio de sus similares en el extranjero" (DE LOPEZ MOREIRA, 2012, p. 48), pues las condiciones de emergencia de centros análogos en Uruguay, Argentina y Brasil habían sido muy diferentes.

La Facultad debió funcionar con serias limitaciones presupuestales. Se organizó en diversas secciones (Letras, Pedagogía, Historia, Matemáticas y Filosofía). Otorgaba títulos de Licenciado y las carreras duraban tres años. El número de estudiantes fue en aumento y se crearon dos posgrados, los doctorados de Historia (1950) y de Filosofía (1951).

El concurso de Guy de Hollanda (1913-1975) fue muy importante en la etapa inicial. Este polifacético intelectual de origen carioca -con sendos doctorados en Derecho e Historia y tecnicaturas en Educación y Bibliotecología- arribó a Paraguay en 1942, luego de haber ejercido la docencia y diversas actividades de carácter cultural en su país natal. Integró la Misión Brasileña y se arraigó fuertemente en el país. Se casó con una paraguaya y permaneció hasta 1956.

Desempeñó una intensa actividad cultural en diversos ámbitos. Fue particularmente destacada su colaboración en el proceso de institucionalización de los estudios humanísticos 
en general y de la formación en Historia en particular. En la Escuela de Humanidades fue docente, entre otras, de una materia clave como Introducción a los Estudios Históricos. Cuando se fundó la Facultad de Filosofía coordinó la carrera de Historia y posteriormente el doctorado. Crónicas de época destacan su afán en la formación de los futuros historiadores a través de una labor teórico-práctica en el Archivo Nacional. Como resultado de sus indagatorias publicó, durante su residencia en Asunción, algunos trabajos interesantes en los que conjugaba la documentación original con la crítica historiográfica. Se destacan en particular, "Los españoles y las castas" (DE HOLLANDA, 1956, p. 69-76) y "Antequera y los comuneros en la historiografía paraguaya" (DE HOLLANDA, 1954, p. 4-6).

La contribución de Guy de Hollanda fue fundamental en el período que Ricardo Pavetti denomina "los tiempos heroicos de la historiografía académica paraguaya" (PAVETTI, 2013, p. 7). En reconocimiento a sus aportes, las autoridades universitarias le otorgaron en 1957 el título de doctor Honoris Causa.

\section{Conclusión}

En la primera mitad del siglo XX se configuraron, en un desarrollo de ritmos variables, los campos historiográficos de los Estados de la Cuenca del Plata. En ese proceso cumplieron un rol importante un conjunto de intelectuales extranjeros que actuaron en centros superiores de formación en Historia. La significación de los mismos varió en cada realidad y estuvo en directa relación a la manera en que se procesó la interfaz entre las novedades que pretendían aportar y las circunstancias objetivas del ámbito de aplicación.

La condiciones epistémicas existentes en Argentina, Brasil y Uruguay posibilitaron una recepción adecuada de las acciones de intercambio, complementariedad y tránsito de influencias teórico-metodológicas gestionadas por Altamira, Braudel, Ravignani y Romero respectivamente. Esos intelectuales debieron insertarse en medios académicos que estaban en proceso de estructuración y actuaron sobre tradiciones historiográficas con diferentes niveles de consolidación. Desempeñaron un rol fundamental en la organización y dinamización de la formación de investigadores profesionales.

La situación de Paraguay fue peculiar. La acción de Guy de Hollanda en la Facultad de Filosofía de la UNA no produjo resultados parangonables con los referidos ut supra. Su magisterio estuvo condicionado por una serie de factores estructurales que ejercieron un 
efecto inercial sobre la indagatoria del pretérito y ocluyeron la implementación de prácticas transformadoras.

Los historiadores extranjeros practicaron, en la mayoría de los casos, un magisterio fecundo sobre las instituciones y los investigadores locales. Este influjo llegó incluso a un público más general a través de la realización de conferencias y de la publicación de artículos en revistas o periódicos locales.

REFERENCIAS BIBLIOGRÁFICAS

AGUIRRE ROJAS, Carlos A. Braudel en las Américas. Ensayo de comparación de dos intercambios transculturales. Signos Históricos. México, II. 3, p. 49-80, 2000.

- La réception de I'historiographie française en Amérique latine. 1870-1968, Caravelle. Toulouse, n. 74, p. 143-158, 2000.

BUARQUE DE HOLANDA, Sérgio. O pensamento histórico no Brasil durante os últimos cinquenta anos. Correio da Manhã. Rio de Janeiro, p. 1-3, 15 de junio de 1951.

BUCHBINDER, Pablo. Historia de las universidades argentinas. Buenos Aires: Sudamericana, 2005.

CORRÊA LIMA, Luís. Fernand Braudel e o Brasil. Vivência e brasilianismo (1935-1945). São Paulo: Edusp, 2009.

DE HOLLANDA, Guy. Antequera y los comuneros en la historiografía paraguaya. Panorama. Arte. Ciencia. Letras. Actualidad. Asunción, año II, n. 14, p. 4-6, 1954.

Los españoles y las castas. Historia Paraguaya. Anuario del Instituto Paraguayo de Investigaciones Históricas. Asunción, n. 1, p. 69-76, 1956.

DE LOPEZ MOREIRA, Mary Monte de (Coordinación general). Evolución histórica de la Facultad de Filosofía de la Universidad Nacional de Asunción. Asunción: UNA, 2012.

DEVOTO, Fernando; PAGANO, Nora. Historia de la historiografía argentina. Buenos Aires: Sudamericana, 2009.

DEVOTO, Fernando J. Itinerario de un problema. Annales y la historiografía argentina (1929-1965). Anuario del IEHS. Tandil, 10, p. 155-175, 1995.

. La historiografía argentina en el siglo XX (Estudio preliminar y compilación). t. I. Buenos Aires: Centro Editor de América Latina, 1993.

D`INCAO, Maria Angela (Org.). História e ideal. Ensaios sobre Caio Prado Junior. São Paulo: Editora Brasiliense, 1989. 
DUARTE, María Amalia. La Escuela Histórica de La Plata. In: ACADEMIA NACIONAL DE LA HISTORIA. La Junta de Historia y Numismática Americana y el movimiento historiográfico en la Argentina (18931938). Buenos Aires: Academia Nacional de la Historia, 1995, t. I, p. 271-294.

FLAMARION CARDOSO, Ciro; VAINFAS, Ronaldo. Domínios da História. Ensaios de Teoria e Metodologia. Rio de Janeiro: Elsevier, 2011, 2 ed.

GAMARRA DOLDÁ, Pedro. Manuel Riquelme, la Facultad de Filosofía y la educación superior. $A B C$ Color. Asunción, p. 2, 4 de mayo de 2014.

GARCÍA, María Amalia, Modelos de internacionalismo y modernidad. Las artes plásticas paraguayas en la encrucijada brasileña. Nuevo Mundo Mundos Nuevos. París, puesto en línea el 11 septiembre 2014. Disponible en: <https://nuevomundo.revues.org/67182>. Acceso el 26 febrero 2016.

JOBSON DE ANDRADE ARRUDA, José. Historia de la Historia en Brasil. Investigaciones Históricas: Época moderna y contemporánea. Valladolid, n. 18, p. 347-384, 1998.

LAUERHASS Ludwig; NAVA Carmen (organizadores). Brasil uma identidade em construção. São Paulo: Atica, 2007.

MARTIRÉ, Eduardo. Altamira y Levene, una amistad provechosa. In: RUBIO CREMADES, Enrique; VALERO JUAN, Eva M. (Eds.). Rafael Altamira: historia, literatura y derecho. Actas del Congreso Internacional celebrado en la Universidad de Alicante, del 10 al 13 de diciembre de 2002. Alicante: Universidad de Alicante, 2004, p. 157-162.

MARTINIÈRE, Guy. La Escuela de los “Annales" y las Américas Latinas (1929 -1949). Estudios Latinoamericanos. Varsovia, 6, p. 133-153, 1980.

PARIS DE ODDONE, Ma. Blanca (Coord.). Historia y memoria. Medio siglo de la Facultad de Humanidades y Ciencias de la Educación. Montevideo: Facultad de Humanidades y Ciencias de la Educación, 1995.

PAVETTI, Ricardo. Prólogo. In: ACOSTA, Gustavo. Posguerra contra la Triple Alianza. Aspectos políticos e institucionales (1870-1904). Asunción: Servilibro, 2013.

PELOSI, Hebe Carmen. Las redes sociales de Rafael Altamira historiador. Canelobre, Alicante, n. 59, p. 47-54, 2012.

Rafael Altamira y la Argentina. Alicante: Universidad de Alicante, 2005.

PRADO, Gustavo. Rafael Altamira en América (1909-1910). Historia e historiografía del proyecto americanista de la Universidad de Oviedo. Madrid: CSIC, 2008.

Rafael Altamira en el Río de la Plata: claves ideológicas e historiográficas de su éxito en la Argentina del Centenario. In: ALTAMIRA, Pilar (Coord.). La Huella de Rafael Altamira. Madrid: Universidad Complutense, 2013, p. 137-153.

REIS, José Carlos. As Identidades do Brasil. De Varnhagen a FHC. Vol. I. 9 edição ampliada. Rio de Janeiro: FGV Editora, 2007.

SIMÕES DE Paula, Eurípedes. Algumas considerações sobre a contribuição da Faculdade de Filosofia, Letras e Ciências Humanas da Universidade de São Paulo para a Historiografia Brasileira. Revista de História. São Paulo, n. 88, p. 425-451, 1971. 
SOZA, Felipe. La historiografía latinoamericana. In: AURELL, Jaume; BALMACEDA, Catalina; BURKE, Peter; SOZA, Felipe. Comprender el pasado. Una historia de la escritura y el pensamiento histórico. Madrid: Akal, 2013, p. 341-437.

TEIXEIRA LOPES, Eliane Marta - MENDES FARIA FILHO, Luciano - GREIVE VEIGA, Cynthia. 500 anos de educação no Brasil. 5 edição. Belo Horizonte: Autêntica, 2015.

ZUBILLAGA, Carlos. Historia e historiadores en el Uruguay del siglo XX. Montevideo: Librería de la Facultad de Humanidades y Ciencias de la Educación, 2002. 